\title{
Analysing instability of combustion waves using the Evans function
}

\author{
J. J. Sharples ${ }^{1} \quad$ H. S. Sidhu ${ }^{2} \quad$ V. V. Gubernov ${ }^{3}$
}

(Received 30 January 2011; revised 21 July 2011)

\begin{abstract}
We consider travelling wave solutions of a reaction-diffusion system corresponding to a single step, homogeneous, premixed combustion scheme with Newtonian heat loss and general Lewis number. Particular attention is paid to unstable combustion wave regimes, especially those associated with oscillatory behaviour. The instability analysis is conducted with the use of Evans function techniques, which we use to derive eigenvalues of the linear stability problem via the argument principle and Nyquist plots. These techniques permit the study of transitions to different modes of unstable behaviour in great detail. Threshold values of the parameters corresponding to Hopf and Bogdanov-Takens bifurcation are established and it is shown that for certain parameter values the system exhibits a period doubling route to chaotic behaviour.
\end{abstract}

http://anziamj . austms . org.au/ojs/index.php/ANZIAMJ/article/view/3944 gives this article, (c) Austral. Mathematical Soc. 2011. Published September 23, 2011. ISSN 1446-8735. (Print two pages per sheet of paper.) Copies of this article must not be made otherwise available on the internet; instead link directly to this URL for this article. 


\section{Contents}

1 Introduction

C774

2 Mathematical model

C776

3 Combustion wave stability

C778

3.1 Evans function . . . . . . . . . . . . . . . . C779

3.2 Compound matrix method . . . . . . . . . . . . C C781

4 Results

C782

4.1 Combustion wave properties . . . . . . . . . . . C C782

4.2 Stability properties . . . . . . . . . . . . C782

5 Discussion

C785

References

C789

\section{Introduction}

Understanding combustion is important for a variety of applications including industrial processes, mining safety, explosives engineering, the synthesis of advanced materials and even environmental problems such as bushfires. As a consequence, models that simulate combustion processes have been studied by researchers over a long period of time [11]. In general, the models used to simulate combustion processes are systems of partial differential equations that account for the reaction and diffusion of heat, reactants and products. As such the study of combustion processes inevitably amounts to the study of reaction-diffusion systems.

The precise chemical reaction mechanism of a particular combustion process is typically extremely complex, often involving tens to hundreds of individual reaction steps. However, to make combustion amenable to mathematical 
analysis it is common to view the overall combustion process as a small number of lumped reaction steps, which are representative of the actual chemical kinetics. For example, much of the progress in developing the theory of combustion has been based on reaction mechanisms involving only one or two steps [3]. In single step reaction schemes it is assumed that the reaction proceeds as a single exothermic reaction between the fuel $(\mathrm{F})$ and the oxidant, herein assumed to be oxygen $\left(\mathrm{O}_{2}\right)$, which combine to produce combustion products $(\mathrm{P})$ and heat. The generic kinetic scheme for a single step reaction mechanism is thus

$$
\mathrm{F}+\mathrm{O}_{2} \longrightarrow \mathrm{P}+\text { heat }
$$

Of interest is the manner in which flames propagate through a particular mixture. As such, there has been considerable interest in reaction-diffusion systems that admit travelling wave solutions, which in the context of combustion, are called combustion waves. A combustion wave describes a propagating flame front, which marks the transition from the initial mixture to the reactantproduct equilibrium phase. Reaction-diffusion systems describing combustion processes are distinguished by the extremely strong nonlinear dependence of the reaction rate on temperature, usually described by the Arrhenius law that the rate

$$
\mathrm{k}(\mathrm{T})=A \exp \left(\frac{-\mathrm{E}}{\mathrm{RT}}\right),
$$

where $A$ is the pre-exponential constant, $E$ is the activation energy, and $T$ is the temperature of the reaction. The universal gas constant is denoted by $R$. The strong nonlinearity that arises through invocation of the Arrhenius law complicates the analysis of combustion waves. Before the advent of modern computing power investigations typically relied on asymptotic methods or advanced numerical techniques.

Of particular interest are the various modes of instability that arise in combustion processes. Combustion instabilities can have undesirable, or even disastrous effects on the safety and efficiency of industrial and synthetic processes [10]; they can seriously compromise the safety of personnel that operate 
in confined or volatile spaces such as compartments or underground mines [15]; and they can produce unexpected and dangerous wildfire behaviour [4]. Recent work on two step reaction schemes revealed that the dynamics of the associated combustion waves can be quite complex. Indeed, Gubernov et al. [9] demonstrated that combustion waves arising in the presence of chain branching kinetics can exhibit oscillatory behaviour and progress through a series of period doublings en route to chaotic behaviour.

We investigate the stability of combustion waves arising under the assumption of a nonadiabatic, single step, reaction mechanism. The effects of heat loss from the system are modelled via the inclusion of a term describing Newtonian cooling. The transition to instability is investigated in great detail using the Evans function method, which permits a significant improvement in accuracy over methods relying on direct integration of the governing reaction-diffusion equations. Evans function techniques can also be applied more generally to investigate the stability of travelling waves arising in applications such as nerve impulse propagation, (non-combustion) reactive waves, transmission and propagation of beams through wave guides, shock waves arising in conservation laws relating to fluid, and gas dynamics and localised vibrations in solids [13]. We aim to elucidate the Evans function method as it applies to the single step non-adiabatic reaction scheme in some detail so as to aid researchers interested in applying the Evans function technique in their own area of interest.

\section{Mathematical model}

Consider flame propagation through a premixed fuel in one spatial dimension with combustion described by the single step reaction mechanism. Additionally, suppose that heat is lost to the surrounding environment through Newtonian cooling. A real world example of such a configuration would be a long, imperfectly insulated cylinder containing a fuel-oxygen mixture, with an appropriate a priori averaging over the transverse spatial dimension of 
the flame front. Assuming that the rate of the exothermic combustion is adequately described by the Arrhenius law, the equations describing conservation of energy and mass lead to the following (non-dimensionalised) reaction-diffusion PDE system [16]:

$$
\begin{aligned}
& u_{\mathrm{t}}=u_{x x}+v e^{-1 / u}-\mathrm{l}\left(\mathrm{u}-\mathrm{u}_{\mathrm{a}}\right), \\
& v_{\mathrm{t}}=\mathrm{Le}^{-1} v_{x x}-\beta v e^{-1 / \mathrm{u}},
\end{aligned}
$$

where $\mathfrak{u}(x, t)$ and $v(x, t)$ are the non-dimensional temperature and mass fraction of fuel, respectively, Le is the Lewis number (the ratio of the diffusion rates of mass and heat), $\beta$ is the ratio of the activation energy to heat release, $l$ is the volumetric heat-loss coefficient from fuel to surroundings, and $u_{a}$ is the non-dimensional ambient temperature. For convenience we assume $\mathfrak{u}_{\mathrm{a}}=0$. This assumption does not affect the qualitative properties of the travelling waves [7] and circumvents the cold boundary problem [16].

The system (1)-(2) is supplemented by the boundary conditions

$$
\begin{array}{lll}
u(x, t)=0, & v(x, t)=\sigma \quad \text { as } x \rightarrow-\infty \\
u(x, t)=0, & v(x, t)=1 \quad \text { as } x \rightarrow+\infty
\end{array}
$$

The conditions on the right boundary $(x \rightarrow+\infty)$ correspond to cold, unburned fuel, while the left boundary conditions $(x \rightarrow-\infty)$ correspond to cold, burnt fuel. We allow for an amount $\sigma$ of unburnt fuel remaining behind the combustion front.

As we are interested in travelling wave solutions, which propagate with speed c we reformulate the system (1)-(4) in terms of the coordinate $\xi=x-c t$, in the frame that moves with the combustion front. We thereby obtain the system of ordinary differential equations

$$
\begin{aligned}
& u_{\xi \xi}+c u_{\xi}+v e^{-1 / u}=l u, \\
& \operatorname{Le}^{-1} v_{\xi \xi}+c v_{\xi}-\beta v e^{-1 / u}=0,
\end{aligned}
$$

with boundary conditions

$$
u(\xi)=0, \quad v(\xi)=\sigma \quad \text { as } \xi \rightarrow-\infty,
$$




$$
u(\xi)=0, \quad v(\xi)=1 \quad \text { as } \xi \rightarrow+\infty
$$

Combustion wave solutions to (5)-(8) were derived numerically over a wide range of parameter values using shooting and relaxation methods as described by Sharples et al. [14].

\section{Combustion wave stability}

Stability of a combustion wave refers to the development of solutions of the governing equations whose initial conditions comprise a perturbation of the combustion wave under consideration. If there are initial conditions arbitrarily close to the combustion wave such that the associated solutions leave a small neighbourhood of the combustion wave, then the wave is unstable. A natural approach to investigating the stability of travelling waves is to linearise the governing partial differential equations about the wave and to study the spectrum of the resulting linear differential operator $\mathcal{L}$. In our case the linearised equations are

$$
\mathbf{U}_{\mathrm{t}}=\mathcal{L} \mathbf{U}
$$

where

$$
\mathcal{L} \mathbf{U}=\mathrm{DU}_{\xi \xi}+\mathrm{c} \mathbf{U}_{\xi}+W \mathbf{U},
$$

with $\mathbf{U}(\xi, \mathrm{t})=(\mathbf{U}(\xi, \mathrm{t}), \mathbf{V}(\xi, \mathrm{t}))^{\mathrm{T}}, \mathrm{D}=\operatorname{diag}\left(1, \mathrm{Le}^{-1}\right)$ and

$$
W=\left(\begin{array}{cc}
v u^{-2} e^{-1 / u}-l & e^{-1 / u} \\
-\beta v u^{-2} e^{-1 / u} & -\beta e^{-1 / u}
\end{array}\right)
$$

where $u=u(\xi)$ and $v=v(\xi)$ are the travelling wave solutions of (5)-(8).

Seeking solutions in the form $\mathbf{U}(\xi, t)=\mathbf{U}(\xi) e^{\lambda t}$ the system (9) may be written as the first order system of ordinary differential equations

$$
\mathbf{z}_{\xi}=\mathbf{A}(\xi, \lambda) \mathbf{z}
$$


where matrix

$$
\mathbf{A}(\xi, \lambda)=\left(\begin{array}{cccc}
0 & 0 & 1 & 0 \\
0 & 0 & 0 & 1 \\
\lambda+\mathrm{l}-v \mathrm{uu}^{-2} e^{-1 / u} & -e^{-1 / u} & -c & 0 \\
\beta \operatorname{Le} v u^{-2} e^{-1 / u} & \operatorname{Le}\left(\lambda+\beta e^{-1 / u}\right) & 0 & -c \text { Le }
\end{array}\right),
$$

and we have set $\mathbf{z}=\left(\mathbf{U}, \mathbf{U}_{\xi}\right)^{\mathrm{T}} \in \mathbb{C}^{4}$. The spectral parameter $\lambda$ is sometimes referred to as the growth rate in the context of mathematical combustion theory. Following Afendikov and Bridges [1], a combustion wave is deemed linearly unstable if, for some fixed eigenvalue $\lambda \in \mathbb{C}$ with $\mathfrak{R} \lambda>0$, there exists a solution of (11) that decays exponentially as $\xi \rightarrow \pm \infty$.

\subsection{Evans function}

Substituting the boundary conditions (7)-(8) into (12) we obtain the limit matrices

$$
\mathbf{A}^{ \pm}(\lambda)=\left(\begin{array}{cccc}
0 & 0 & 1 & 0 \\
0 & 0 & 0 & 1 \\
\lambda+l & 0 & -c & 0 \\
0 & \lambda \text { Le } & 0 & -c \text { Le }
\end{array}\right)
$$

which have eigenvalues

$$
\begin{aligned}
& \mu_{1}^{ \pm}(\lambda)=\frac{1}{2}\left(-c \operatorname{Le}-\sqrt{c^{2} L^{2}+4 \lambda L e}\right), \\
& \mu_{2}^{ \pm}(\lambda)=\frac{1}{2}\left(-c \operatorname{Le}+\sqrt{c^{2} L^{2}+4 \lambda L e}\right), \\
& \mu_{3}^{ \pm}(\lambda)=\frac{1}{2}\left(-c-\sqrt{c^{2}+4(\lambda+l)}\right), \\
& \mu_{4}^{ \pm}(\lambda)=\frac{1}{2}\left(-c+\sqrt{c^{2}+4(\lambda+l)}\right),
\end{aligned}
$$

and corresponding eigenvectors $\mathbf{k}_{i}^{ \pm}(\lambda), i=1, \ldots, 4$.

Assuming that $\mathfrak{R} \lambda>0$, it follows that there are two linearly independent solutions $\mathbf{z}_{2,4}^{-}(\xi, \lambda)$ which span the eigenspace $E^{-}(\xi, \lambda)$ of solutions that decay 
exponentially as $\xi \rightarrow-\infty$ and two linearly independent solutions $\mathbf{z}_{1,3}^{+}(\xi, \lambda)$ which span the eigenspace $E^{+}(\xi, \lambda)$ of solutions that decay exponentially as $\xi \rightarrow+\infty$. If $E^{-}(\xi, \lambda)$ and $E^{+}(\xi, \lambda)$ have nontrivial intersection for some value of $\lambda$, then $\lambda$ is an eigenvalue.

Denoting the space of two-forms over $\mathbb{C}^{4}$ as $\Lambda^{2}\left(\mathbb{C}^{4}\right)$, we define $\mathbf{V}^{ \pm} \in \Lambda^{2}\left(\mathbb{C}^{4}\right)$ as the exterior products

$$
\mathbf{V}^{-}=\mathbf{z}_{2}^{-} \wedge \mathbf{z}_{4}^{-} \quad \text { and } \quad \mathbf{V}^{+}=\mathbf{z}_{1}^{+} \wedge \mathbf{z}_{3}^{+} .
$$

Hence the eigenspaces $\mathrm{E}^{-}(\xi, \lambda)$ and $\mathrm{E}^{+}(\xi, \lambda)$ intersect nontrivially at a certain value of $\xi$ if and only if $\mathbf{V}^{-}(\xi, \lambda) \wedge \mathbf{V}^{+}(\xi, \lambda)=0$. We take $\xi=0$ for definiteness. The foregoing discussion motivates the definition of the Evans function $[2,6]$ :

$$
\widetilde{\mathrm{D}}(\lambda)=\mathbf{V}^{-}(0, \lambda) \wedge \mathbf{V}^{+}(0, \lambda) .
$$

Moreover, if $\left\{\mathbf{e}_{1}, \mathbf{e}_{2}, \mathbf{e}_{3}, \mathbf{e}_{4}\right\}$ is a basis in $\mathbb{C}^{4}$, then the canonical isomorphism between $\mathbb{C}^{4}$ and $\Lambda^{4}\left(\mathbb{C}^{4}\right)$ implies that there exists $D(\lambda) \in \mathbb{C}$ such that

$$
\widetilde{\mathrm{D}}(\lambda)=\mathrm{D}(\lambda) \mathbf{e}_{1} \wedge \mathbf{e}_{2} \wedge \mathbf{e}_{3} \wedge \mathbf{e}_{4} .
$$

In terms of the natural basis for $\Lambda^{2}\left(\mathbb{C}^{4}\right)$,

$$
\begin{aligned}
& \mathbf{v}_{1}=\mathbf{e}_{1} \wedge \mathbf{e}_{2}, \quad \mathbf{v}_{2}=\mathbf{e}_{1} \wedge \mathbf{e}_{3}, \quad \mathbf{v}_{3}=\mathbf{e}_{1} \wedge \mathbf{e}_{4}, \\
& \mathbf{v}_{4}=\mathbf{e}_{2} \wedge \mathbf{e}_{3}, \quad \mathbf{v}_{5}=\mathbf{e}_{2} \wedge \mathbf{e}_{4}, \quad \mathbf{v}_{6}=\mathbf{e}_{3} \wedge \mathbf{e}_{4},
\end{aligned}
$$

it can be shown that [1]:

$$
\mathrm{D}(\lambda)=\mathbf{V}_{1}^{+} \mathbf{V}_{6}^{-}-\mathbf{V}_{2}^{+} \mathbf{V}_{5}^{-}+\mathbf{V}_{3}^{+} \mathbf{V}_{4}^{-}+\mathbf{V}_{4}^{+} \mathbf{V}_{3}^{-}-\mathbf{V}_{5}^{+} \mathbf{V}_{2}^{-}+\mathbf{V}_{6}^{+} \mathbf{V}_{1}^{-} .
$$

As indicated above, the Evans function D has zeroes that coincide with eigenvalues of the linear stability problem. It can also be shown that $\mathrm{D}$ is analytic over $\Omega=\{w \in \mathbb{C}: \mathfrak{R} w \geqslant 0\}$, is asymptotically constant and that $\mathrm{D}(\mathbb{R}) \subset \mathbb{R}$. These properties mean that the problem of finding eigenvalues of 
the linearised operator $\mathcal{L}$ is reduced to one of finding zeroes of an analytic function. Using the argument principle, applied to an analytic function,

$$
\oint_{C} \frac{\mathrm{D}^{\prime}(w)}{\mathrm{D}(w)} \mathrm{d} w=2 \pi \mathrm{iN}
$$

where $\mathrm{N}$ is the number of zeroes of $\mathrm{D}$ within the closed circuit $\mathrm{C}$, and the right-hand side of the equation represents the change in argument of $\mathrm{D}(w)$ as $C$ is traversed. Hence taking $C$ to be the imaginary axis $\{i t: t \in \mathbb{R}\}$ the number of zeroes of $\mathrm{D}$ in the right complex half-plane is simply the number of times $\mathrm{D}(\mathrm{it})$ winds around the origin.

Our attention now turns to the numerical method for calculating the Evans function D.

\subsection{Compound matrix method}

Consider the two-form $\mathbf{V}=\mathbf{z}_{1} \wedge \mathbf{z}_{2}$, where $\mathbf{z}_{1}$ and $\mathbf{z}_{2}$ are solutions of (11). It can be shown that $\mathbf{V}$ satisfies the compound matrix problem [8]:

$$
\mathbf{V}_{\xi}=\mathbf{B}(\xi, \lambda) \mathbf{V},
$$

where

$$
\begin{aligned}
{[\mathbf{B}(\xi, \lambda) \mathbf{V}]_{1}=} & \mathbf{V}_{3}-\mathbf{V}_{4}, \\
{[\mathbf{B}(\xi, \lambda) \mathbf{V}]_{2}=} & -e^{-1 / u} \mathbf{V}_{1}-\mathbf{c V}_{2}, \\
{[\mathbf{B}(\xi, \lambda) \mathbf{V}]_{3}=} & \operatorname{Le}\left(\lambda+\beta e^{-1 / u}\right) \mathbf{V}_{1}-c \operatorname{Le} \mathbf{V}_{2}+\mathbf{V}_{6}, \\
{[\mathbf{B}(\xi, \lambda) \mathbf{V}]_{4}=} & \left(v u^{-2} e^{-1 / u}-\lambda-l\right) \mathbf{V}_{1}-\mathbf{c V}_{4}-\mathbf{V}_{6}, \\
{[\mathbf{B}(\xi, \lambda) \mathbf{V}]_{5}=} & -\operatorname{Le} \beta v u^{-2} e^{-1 / u} \mathbf{V}_{1}-c \operatorname{Le} \mathbf{V}_{5} \\
{[\mathbf{B}(\xi, \lambda) \mathbf{V}]_{6}=} & -\operatorname{Le} \beta v u^{-2} e^{-1 / u} \mathbf{V}_{2}+\left(\lambda+l-v u^{-2} e^{-1 / u}\right) \mathbf{V}_{3} \\
& -\operatorname{Le}\left(\lambda+\beta e^{-1 / u}\right) \mathbf{V}_{4}-e^{-1 / u} \mathbf{V}_{5}-(c+c \operatorname{Le}) \mathbf{V}_{6},
\end{aligned}
$$

and where $u=u(\xi)$ and $v=v(\xi)$ again denote the travelling wave solution of (5)-(8). 
It can also be shown that $\mathbf{V}^{-}(\xi)$, defined by (18) is the solution of (24) with the largest rate of exponential growth, $\mu_{2}^{-}+\mu_{4}^{-}$, integrating forward from $\xi=-\infty$ to $\xi=0$. Similarly, $\mathbf{V}^{+}(\xi)$ defined by (18) is the solution of (24) with the largest rate of exponential growth, $\mu_{1}^{+}+\mu_{3}^{+}$, integrating backward from $\xi=+\infty$ to $\xi=0$. Thus the Evans function is evaluated via equation (22) using standard tools for numerical integration, while avoiding the numerical difficulties usually encountered when dealing with stiff systems [6, 12].

\section{Results}

\subsection{Combustion wave properties}

Equations (5)-(8) are solved numerically using shooting and relaxation methods [6]. Figure 1 illustrates how the combustion wave speed $c$ varies with the parameter $\beta$, for the two cases Le $=5.0$ and $l=1 \times 10^{-4}$ (Figure $1(\mathrm{a})$ ) and $l=2 \times 10^{-4}$ (Figure 1(b)). The curves in Figure 1 are double valued with a turning point (fold bifurcation), which is sometimes referred to as the extinction point. Three distinct solution branches are depicted in Figure 1: a stable branch (black) corresponding to stable, constant speed combustion waves; an oscillatory unstable branch (red); and uniformly unstable branch (blue). Other properties of the combustion waves, such as maximum temperature and the residual fuel remaining behind the combustion front, may be obtained using the same methods used to derive the wave speeds $[6,7]$.

\subsection{Stability properties}

The stability properties of the combustion waves are derived numerically using the Evans function techniques described above. Figure 2 shows images of the Evans function $\{\mathrm{D}(\mathrm{it}): t \geqslant 0\}$, corresponding to $\mathrm{Le}=5.0$ and $l=1 \times 10^{-4}$ for three different values of $\beta$. Note that only the images of the positive 


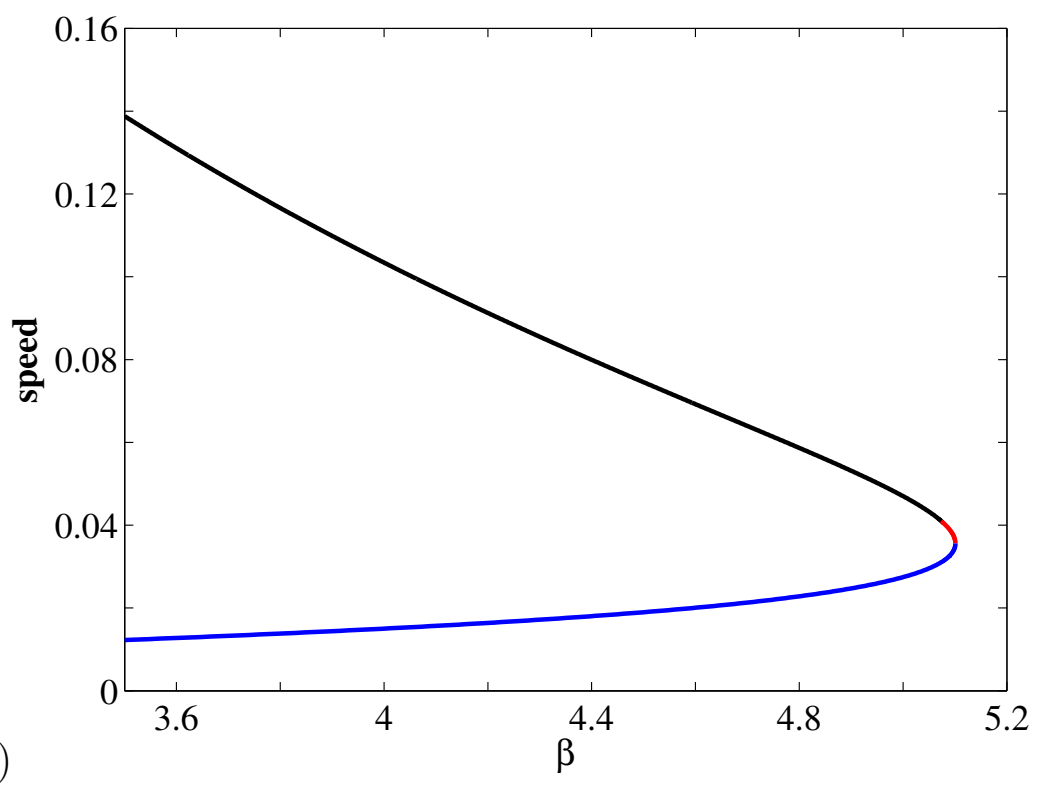

(a)

$\beta$

(b)

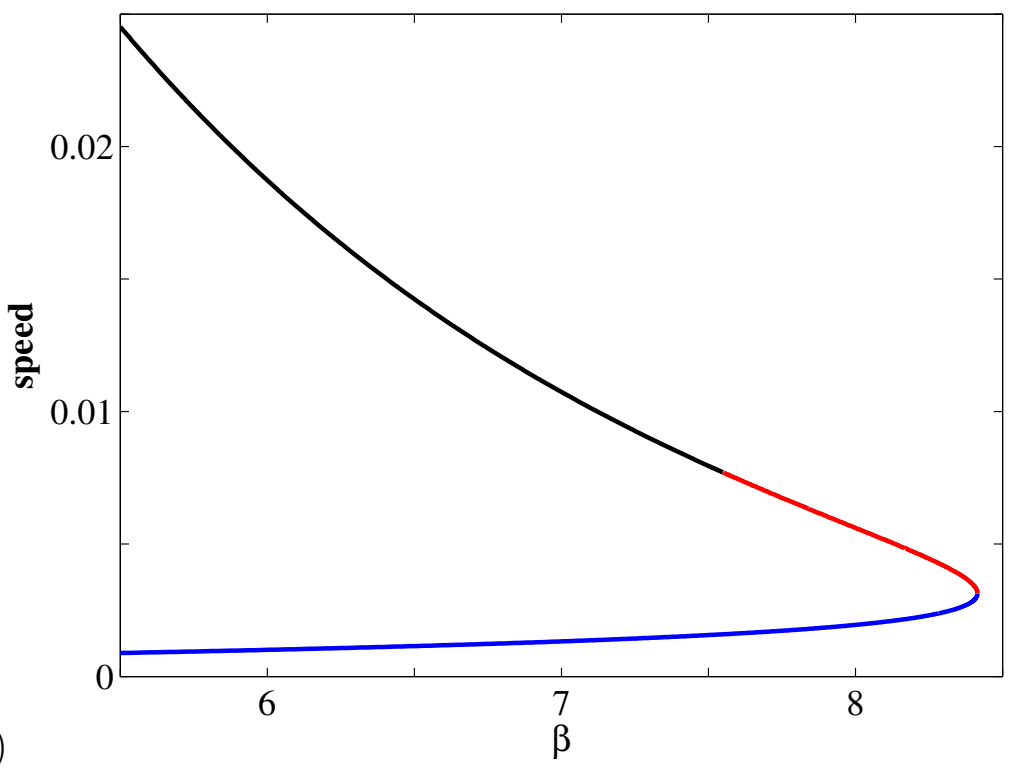

Figure 1: Combustion wave speed plotted against $\beta$ for (a) Le $=5.0$ and $l=1 \times 10^{-4}$; (b) Le $=5.0$ and $l=2 \times 10^{-4}$. 


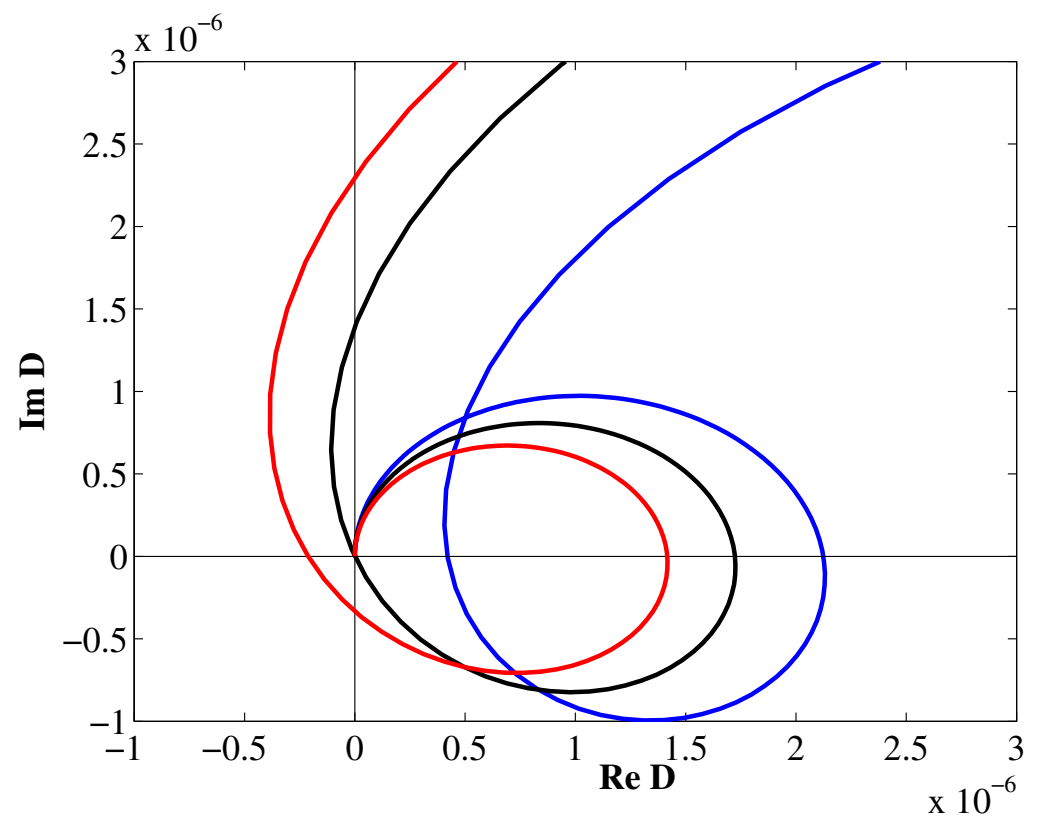

Figure 2: Evans function image curves $\{\mathrm{D}(\mathrm{it}): \mathrm{t} \geqslant 0\}$, corresponding to $\mathrm{Le}=$ 5.0 and $l=1 \times 10^{-4}$, for the three cases $\beta=5.07$ (blue), 5.076465 (black), and 5.08 (red).

imaginary axis is shown for clarity. The images of the negative imaginary axis are simply the complex conjugates of the curves shown in Figure 2, due to $\mathrm{D}(\bar{\lambda})=\overline{\mathrm{D}(\lambda)}$, for all $\lambda \in \mathbb{C}$.

The blue curve, corresponding to $\beta=5.07$, does not encircle the origin and so by the argument principle, the Evans function does not possess any zeroes in the right half of the complex plane. Hence $\beta=5.07$ represents a stable combustion wave. Conversely, the red curve corresponding to $\beta=5.08$, does encircle the origin, which means that the Evans function does have a zero with positive real part, which is an eigenvalue of the linear stability problem. Hence $\beta=5.08$ represents an unstable combustion wave. Application of the argument principle with a closed curve (e.g., a rectangle) that omits the 
positive real axis confirms that for $\beta=5.08$ the zero of the Evans function is of the form $\lambda=\eta+i \omega$ with $\omega>0$ and so the corresponding combustion wave is oscillatory unstable. That is, the combustion wave will only be physically discernable as a combustion front whose speed varies periodicallythe frequency of the oscillation is $\omega$. The black curve in Figure 2 corresponds to the Hopf point $\beta_{\mathrm{h}}=5.076465$ which marks the transistion between the stable, constant speed combustion wave regime and the oscillatory unstable combustion wave regime (Figure 1(a)).

The oscillatory unstable combustion waves are further investigated using a numerical finite element package [5] to solve the partial differential equations (1)-(4) with $u_{a}=0$. For the case Le $=5.0$ and $l=1 \times 10^{-4}$ only period one, pulsating instabilities are detectable. However, for the case $l=2 \times 10^{-4}$ (Figure 1(b)) a number of different oscillatory regimes are found. Indeed, as $\beta$ increases beyond the Hopf point the combustion waves exhibit a sequence of period doublings. Figures 3-4 show the variation in the speed of the combustion front over time corresponding to several values of $\beta$ greater than the Hopf point $\beta_{\mathrm{h}} \simeq 7.55$. Figure 3(a) illustrates period one oscillations at $\beta=7.7$, while Figures $3(\mathrm{~b})$ and $4(\mathrm{a})$ show period two and period four oscillations corresponding to $\beta=7.9$ and $\beta=7.91$, respectively. Assuming a value of $\beta=7.912$ yields an essentially chaotic combustion wave speed, as evident in Figure 4(b). Beyond this value of $\beta$ no sustained combustion front solutions are obtained.

\section{Discussion}

We presented an analysis of the stability of travelling waves arising in a single step combustion scheme. Stability of the combustion waves was investigated using Evans function techniques, which convert the problem of solving the linearised eigenvalue problem to the much more tractable problem of finding the zeroes of a holomorphic function. To decide whether a particular combustion wave is stable or not, it is not necessary to locate the zeroes of 


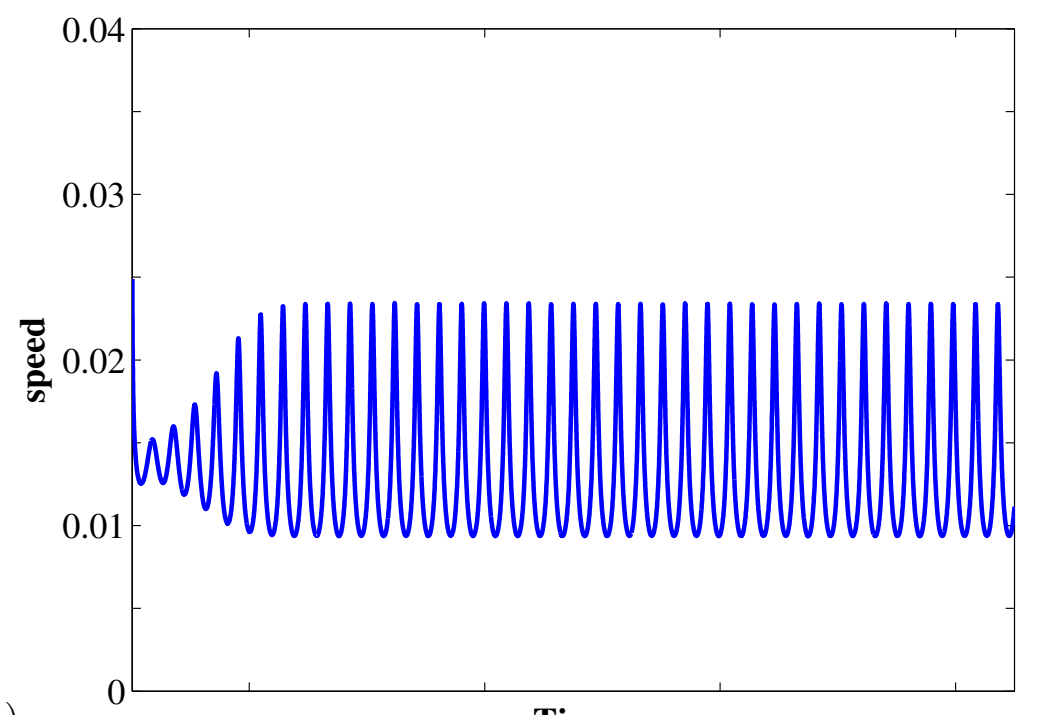

(a)

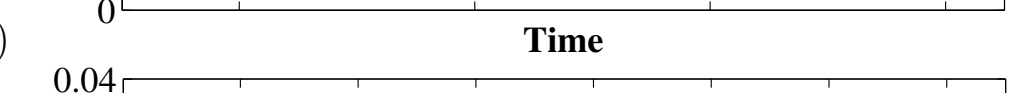

(b)

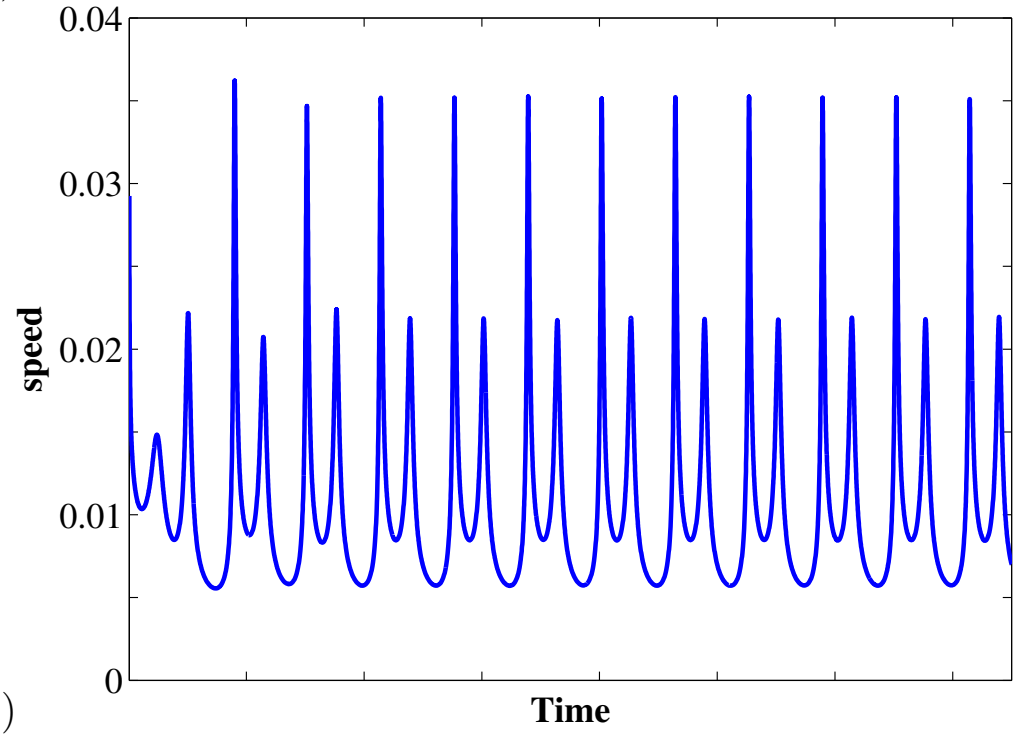

Figure 3: Oscillatory combustion wave regimes for Le $=5.0$ and $l=2 \times 10^{-4}$ : (a) period one oscillations at $\beta=7.7$; (b) period two oscillations at $\beta=7.9$. 


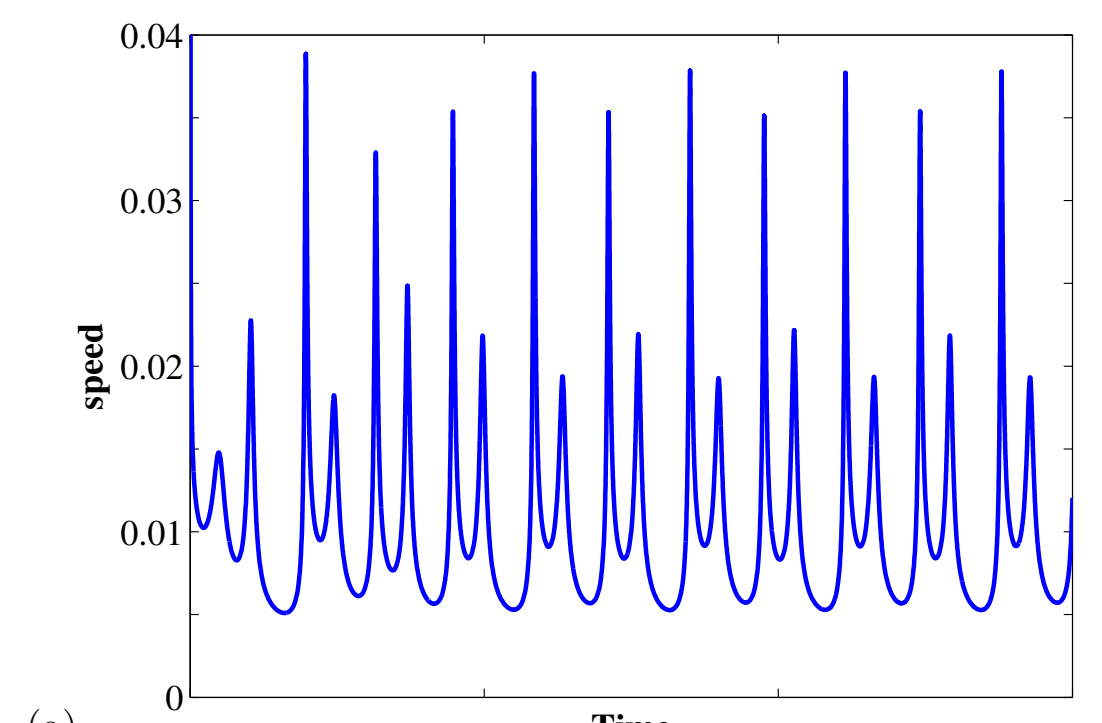

(a)

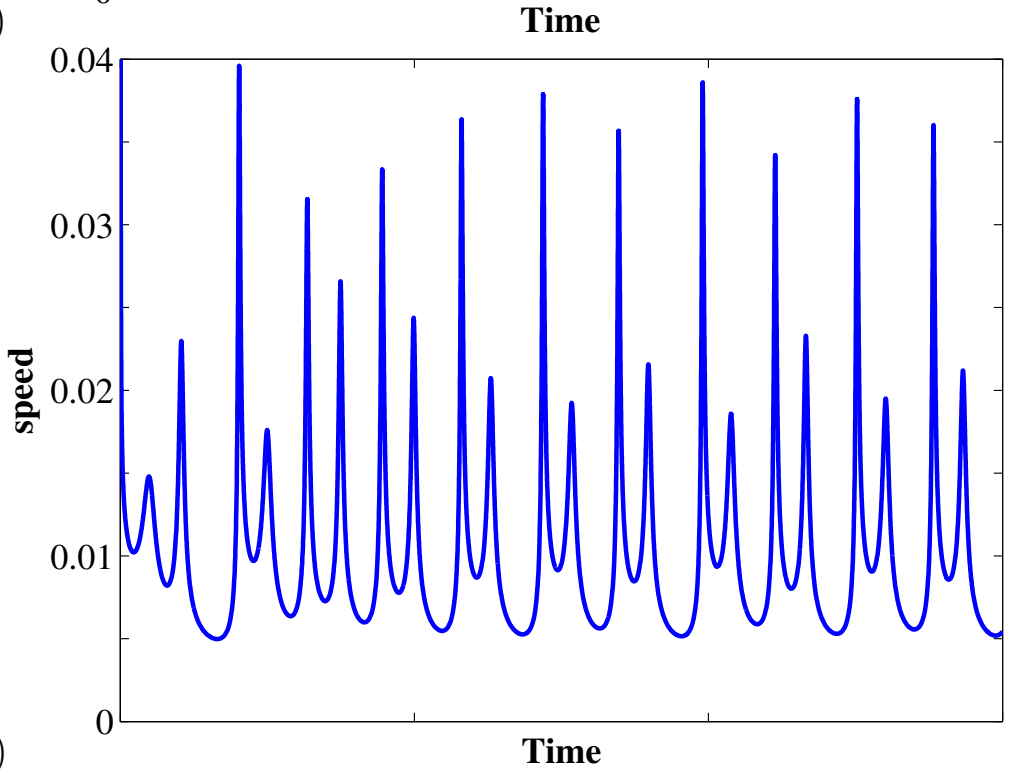

Figure 4: Oscillatory combustion wave regimes for Le $=5.0$ and $l=2 \times 10^{-4}$ : (a) period four oscillations at $\beta=7.91$; and (b) chaotic oscillations at $\beta=7.912$. 
the Evans function explicitly; instead the argument principle simply counts the number of zeroes of the Evans function in the right half-plane. If there are no such zeroes the combustion wave is stable, otherwise the combustion wave is unstable. Simple schemes implementing the argument principle also determine if an unstable combustion wave is uniformly unstable (for which the zero of the Evans function is purely real) or if it possesses an oscillatory instability (for which the zero of the Evans function has non-zero imaginary part).

Stability analysis of combustion schemes, with their strong nonlinear dependence on temperature that arises through the assumption of Arrhenius kinetics, encounter numerical difficulties typically associated with stiff differential equations. However, implementing Evans function techniques via the compound matrix formulation provides a natural way of circumventing these difficulties.

The single step combustion scheme used to exemplify the use of the Evans function has interesting properties that still need to be investigated. This is despite the relatively simple nature of this reaction-diffusion scheme and a long history of the theory of mathematical combustion. For example, for the case $\mathrm{Le}=5.0$ and $l=2 \times 10^{-4}$ the system exhibited a period doubling route to chaotic behaviour, but no such behaviour was found for the case $\mathrm{Le}=5.0$ and $l=1 \times 10^{-4}$. We do not know whether this is due to deficiencies in the methods used to solve the equations numerically, or if it reflects the inherent dynamics of the system. Investigating these aspects of the single step scheme will be the subject of further work. In the meantime we hope that the methods illustrated above will benefit readers interested in the stability analysis of stiff reaction-diffusion systems.

Acknowledgements This work was supported by a grant from the Australian Research Council (DP0878146). 


\section{References}

[1] Afendikov, A. L. and Bridges, T. J. Instability of the Hocking-Stewartson pulse and its implications for three-dimensional Poiseuille flow. Proc. Roy. Soc. A, 457:1-16, 2001. doi:10.1098/rspa.2000.0665 C779, C780

[2] Alexander, J., Gardner, R. and Jones, C. A topological invariant arising in the stability analysis of travelling waves. J. Reine Angew. Math., 410:167-212, 1990. doi:10.1515/crll.1990.410.167 C780

[3] Dold, J. W. Premixed flames modelled with thermally sensitive intermediate branching kinetics. Combust. Theor. Model., 11:909-948, 2007. doi:10.1080/13647830701294599 C775

[4] Dold, J. W., and Zinoviev, A. Fire eruption through intensity and spread rate interaction mediated by flow attachment. Combust. Theor. Model., 13:763-793, 2009. doi:10.1080/13647830902977570 C776

[5] FlexPDE. http://www.pdesolutions.com C785

[6] Gubernov, V. V., Mercer, G. N., Sidhu, H. S. and Weber, R. O. Evans function stability of combustion waves. SIAM J. Appl. Math., 63:1259-1275, 2003. doi:10.1137/S0036139901400240 C780, C782

[7] Gubernov, V. V., Mercer, G. N., Sidhu, H. S. and Weber, R. O. Evans function stability of non-adiabatic combustion waves. P. R. Soc. A, 460:2415-2435, 2004. doi:10.1098/rspa.2004.1285 C777, C782

[8] Gubernov, V. V., Sidhu, H. S. and Mercer, G. N.. Generalized compound matrix method. Appl. Math. Lett., 19:458-463, 2006. doi:10.1016/j.aml.2005.07.002 C781

[9] Gubernov, V. V, Kolobov, A. V., Polezhaev, A. A., Sidhu, H. S. and Mercer, G. N. Period doubling and chaotic transient in a model of 
chain-branching combustion wave propagation. Proc. Roy. Soc. Lond. A, 466:2747-2769, 2010. doi:10.1098/rspa.2009.0668 C776

[10] Makino, A. Fundamental aspects of the heterogeneous flame in the self-propagating high-temperature synthesis (SHS) process. Prog. Energ. Combust., 27:1-74, 2001. doi:10.1016/S0360-1285(00)00004-6 C775

[11] Merzhanov, A. G., and Rumanov, E. N. Physics of reaction waves. Rev. Mod. Phys., 71:1173-1211, 1999. doi:10.1103/RevModPhys.71.1173 $\mathrm{C} 774$

[12] Pego, R. L., Smereka, P. and Weinstein, M. I. Oscillatory instability of travelling waves for a KdV-Burgers equation. Physica D, 67:45-65, 1993. doi:10.1016/0167-2789(93)90197-9 C782

[13] Sandstede, B. Stability of travelling waves. In B. Fiedler, editor, Handbook of Dynamical Systems II, pp. 983-1055. Elsevier, 2002. doi:10.1016/S1874-575X(02)80039-X C776

[14] Sharples, J. J., Sidhu, H. S. and Gubernov, V. V. Properties of nonadiabatic premixed combustion fronts arising in single-step reaction schemes. In Proceedings of Chemeca 2010, Paper No. 357, 26-29 September 2010, Adelaide. ISBN 9780858259713. http://search.informit.com.au/documentSummary; dn= 979163620766769; res=IELENG C778

[15] Thomas, P. H., Bullen, M. L., Quintiere, J. G. and McCaffrey, B. J. Flashover and instabilities in fire behaviour. Combust. Flame, 38:159-171, 1980. doi:10.1016/0010-2180(80)90048-6 C776

[16] Weber, R. O., G. N. Mercer, H. S. Sidhu, and Gray, B. F. Combustion waves for gases $(\mathrm{Le}=1)$ and solids $(\mathrm{Le}=\infty)$. Proc. Roy. Soc. Lond. A, 453:1105-1118, 1997. doi:10.1098/rspa.1997.0062 C777 


\section{Author addresses}

1. J. J. Sharples, Applied and Industrial Mathematics Research Group, School of Physical, Environmental and Mathematical Sciences, University of New South Wales at the Australian Defence Force Academy, Canberra, ACT 2600, Australia.

mailto: j.sharples@adfa.edu .au

2. H. S. Sidhu, Applied and Industrial Mathematics Research Group, School of Physical, Environmental and Mathematical Sciences, University of New South Wales at the Australian Defence Force Academy, Canberra, ACT 2600, Australia.

3. V. V. Gubernov, I.E. Tamm Theory Department, P.N. Lebedev Physical Institute of Russian Academy of Science, Moscow 119991, Russian Federation. 